\title{
Flow Paths: A Standalone Tangible Board System to Create Educational Games
}

\author{
http://dx.doi.org/10.3991/ijet.v10i4.4640 \\ A. Barredo, P. Garaizar \\ Universirty of Deusto, Bilbao, Spain
}

\begin{abstract}
Educational mobile apps are ubiquitous these days. Despite of their huge commercial success, parents and children realized that physical objects manipulation requires a different set of skills, often developed using traditional toys. A century ago, Montessori proposed a methodology that emphasizes the use of physical materials to facilitate self-learning of abstract concepts. Selfcorrecting Montessori's materials allow learners to improve autonomously in a structured environment. In the same vein, Flow Paths provides a standalone tangible board system to create educational games.
\end{abstract}

Index Terms-educational programs, educational technology, tangible user interface, education, games

\section{INTRODUCTION}

In the last five years, millions of educational games have been published in mobile application stores such as Google Play or Apple App Store. Shortly after using them, parents and children realized that physical objects manipulation requires a different set of skills. A century ago, Montessori proposed a methodology that emphasizes the use of physical materials to facilitate self-learning of abstract concepts [1, 2]. Self-correcting Montessori's materials allow learners to improve autonomously in a structured environment. Following a similar approach, Flow Paths provides a standalone tangible board system to create educational games.

Interactive tangible systems are not new. During the last decades there has been a technological evolution in the field of Tangible User Interfaces (TUIs), from digitally augmented paper to physical objects as icons (phicons), manipulatives, or digital sensors / probes [3, 4]. However, many of these proposals (Phidgets [5], TagTiles [6], multi-touch boards [7], or projector-based augmented reality [8]) require a high initial investment. By contrast, Flow Paths uses inexpensive chips and microcontrollers for its board and passive blocks which can be handled without care by non-expert leaners.

Flow Paths is a system to create interactive block-based educational games. Unlike other tangible solutions, Flows Paths do not rely on additional technology such as cameras, video projectors or mobile devices. The Flow Path's board consists of a matrix of connection slots managed via an Arduino microcontroller capable of sending and receiving information to the blocks connected to it. Using i-shaped and 1-shaped blocks, players can create flows from one point (flow source) to another point (sink or terminator). Each block has a multicolor LED that indicates whether it is stacked properly and if it is within the flow or not. The cover story, goals, and difficulty level of educational games made with this block-based system can be redefined dynamically. Players can replace scenario sheets provided with RFID tags to suit their interests and learning needs.

\section{ELECTRONIC COMPONENTS}

Flow Paths relies on well-known a widely used electronic components.

Arduino Uno board. The Arduino Uno is a microcontroller board based on the ATmega328. It provides 14 digital input/output pins (6 of which can be used as PWM outputs), 6 analog inputs, a $16 \mathrm{MHz}$ ceramic resonator, a USB connection, a power jack, an ICSP header, and a reset button. The board can be connected to a computer with a USB cable or powered with a AC-to-DC adapter or battery to be used. The Uno differs from all preceding Arduino boards in its lack of FTDI USB-to-serial driver chip. Instead, it features the Atmega16U2 (Atmega8U2 up to version R2) programmed as a USB-to-serial converter.

MM74HC151 Multiplexers. The MM74HC151 high speed Digital multiplexer takes advantage from advanced silicon-gate CMOS technology. The high noise immunity and low power dissipation of standard CMOS integrated circuits are two of its main features. Moreover, it has the ability to handle 10 LS-TTL loads. The MM74HC151 selects one of the 8 data sources depending on the address presented on the $\mathrm{A}, \mathrm{B}$, and $\mathrm{C}$ inputs. It features both true (Y) and complement (W) outputs. The STROBE input must be at a low logic level to enable this multiplexer. A high logic level at the STROBE input forces the W output to be HIGH and the Y output to be LOW.

SM130 Mifare RFID reader. The SM130 is a double layer, 28 pin DIP type module that is integrated with a microcontroller, analog \& digital signal processor, IC, and all necessary passive components on both top and bottom layer to complete a fully functional ISO14443A Mifare Classic read/write module.

MCP $23 X 17$ port expander. The MCP23X17 provides multiple 8-bit configuration registers for input, output and polarity selection. The system master can enable the $\mathrm{I} / \mathrm{O}$ ports as inputs or outputs by setting the I/O configuration bits (IODIRA/B). Data for each input or output is kept in the corresponding input or output register. The polarity of the Input Port register can be inverted with the Polarity Inversion register. All registers can be read by the system's master.

Communications. All Flows Paths' components are connected through the I2C protocol to control and gather data from the RFID reader, and through a simple 6-bit protocol to define the selections of the multiplexers. 
Blocks. Regardless their final use and shape, all blocks use the same technology. Each block has a RFID tag on the bottom. Once it is plugged into the board, the data of the RFID tag is transferred to the Arduino microcontroller and then conveniently processed.

Antennas. Four round cooper antennas are placed below each functional position of the board. They are powered by the Arduino board and managed by the multiplexers.

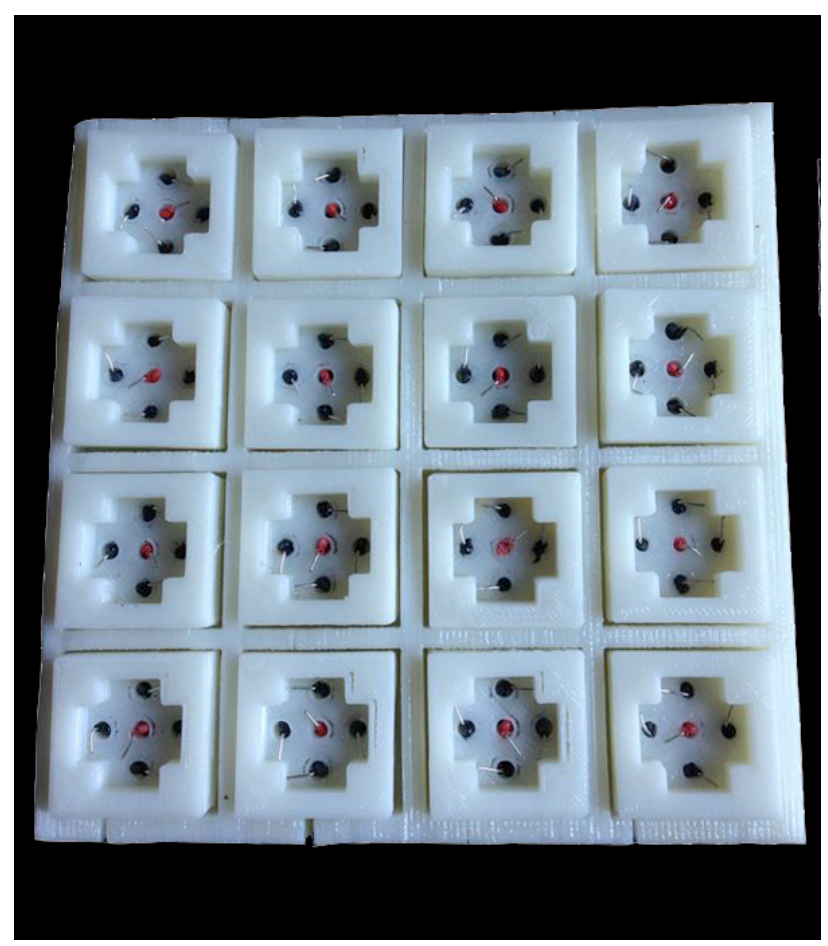

Figure 1. A Flow Paths board ( $4 \times 4$ slots).

\section{TECHNOLOGICAL DETAILS}

Technologically, Flow Paths consists of a board, blocks, and scenario sheets. There is no need to use a computer or mobile device to play with Flow Paths, it is a standalone tangible board system.

As mentioned before, inside Flow Paths' board there is an Arduino Uno board to manage the events of the game and the communication and control of the other electronic devices. Taking into account that we are trying to make a standalone tool, the Arduino Uno board was chosen among others due to its low consumption and nonexpensive price. This board works with an open source version of $\mathrm{C}$ programming language toolchain. Along with its versatile functions, the Arduino Uno also allows communicating different boards between them. This means that the road to link several boards to make bigger and interoperable boards is still open.

Multiplexers are meant to control the RFID antennas accessed by an RFID reader. The RFID reader used in Flow Paths board (Mifare SM130) is a $33.56 \mathrm{Mhz}$ reader. We chose this reader because its wide range reading RFID tags. This way, the number of readers needed to compose the board is reduced (see Fig. 1).

The blocks used in Flow Paths are different depending on who is using them. In the next section two examples of learning games based on Flow Paths are explained. Students at early stages need more visual hints than higher education students. In the case of the game designed for early-stages students, there are two types of blocks: straight paths -I-shaped blocks- and curved paths -Lshaped blocks- (see Fig. 2). Both are implemented in the same way: every block has a RFID tag at the bottom to transmit to the board the information about the block that has been placed into the slot. Moreover, blocks for earlystages students are labeled with numbers and letters for easy identification. Conversely, blocks designed for higher-education students are labeled with fake serial numbers mimicking the identification of electronics components. However, they work on the same principle as the blocks of the game designed for early-stages: an RFID tag placed at the bottom to be read by the Flow Paths board.

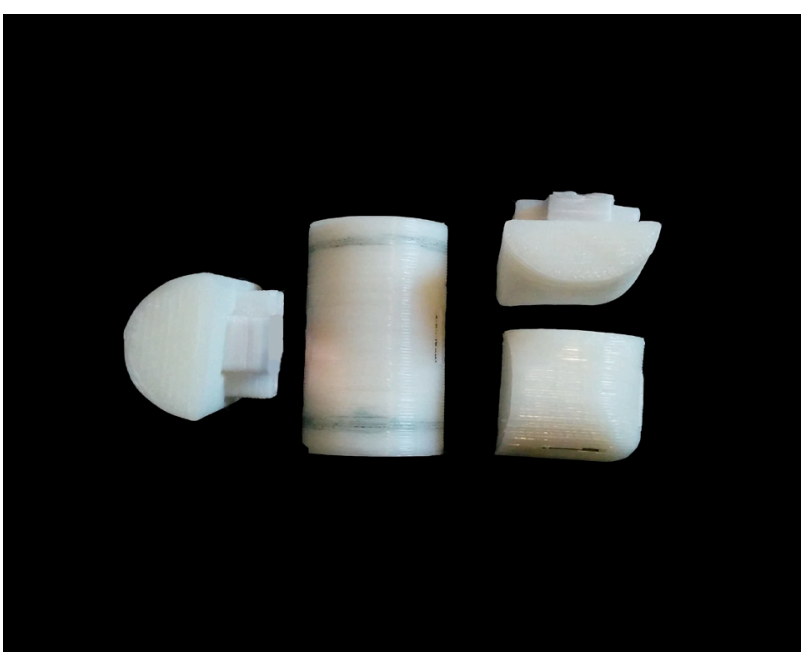

Figure 2. Flow Paths blocks (top and front views).

Reading the RFID tags of the blocks placed on the slots of the board, the Arduino Uno microcontroller is able to gather the layout of all blocks and store it in memory (using an array of blocks' positions and shapes). Once detected the RFID tag of the scenario sheet, the Flow Paths board can apply the logic of the game to the blocks layout and activate the LEDs of the blocks consequently (e.g., in the case of the water pipes game, the pipe-shaped blocks create a water flow which is illuminated in blue, while the remaining blocks are illuminated in red to indicate that they are placed incorrectly).

Through the use of game sheets Flow Paths games can be adapted to any context and level of difficulty by users, developers, or third-parties. The only requirement to create a new game sheet is to use a RFID tag compatible with the SM130 Mifare RFID reader placed in the Flow Paths board. Then, game designers can define the scenario and goal of the game, whereas developers adapt the way the Arduino Uno board interacts with the blocks placed on the board. Therefore, it offers a wide range of new educational opportunities. In the following section, we explain two of them.

\section{SELF-LEARNING OPPORTUNITIES}

Flow Paths pretends to be a system to create new games easily. To accomplish this, it provides a standalone board that can be programmed adapting the source code examples to each need. Flows Paths behavior can be changed using sheets. These sheets are provided with a RFID tag and a background image to provide enough contexts about the goals of the game. Developers can 
adapt provided examples with no effort because all of them follow the same pattern.

Example 1: Water flows. This game is designed to show how the water flows in nature. Young kids are able to interact with the blocks in order to better understand how spatial configuration and gravity can affect water flows.

The sheet of this game shows a cloudy sky on the top and a mountain near to the sea on the bottom (see Fig. 3). On the right side, the sheet provides a brief explanation about the goals of this level (i.e., each Flow Paths game consists in a set of levels with different goals and complexity), the number of blocks meant to be used, and the slots of the board that should be used to solve it. Flows Paths blocks aimed to be used by kids have numbers written on their back. The purpose of numbering the blocks is twofold. First, it is easier to define which blocks should be used to solve a challenge in a Flows Paths game (e.g., explicit indications: "you should use blocks 1, 3, 5, and 4"). Second, game designers can provide subtle hints about the blocks involved in the solution of a challenge in a Flow Paths game (e.g., implicit indications: "decompose these numbers to know which blocks are involved: 13,24 , and 35").

When players use incorrect blocks, they will be provided with instant feedback, as we explain later. Similarly, each slot on the board is also labeled with a letter, and therefore can also be used to provide hints to gamers (e.g., "Hint: start placing the first block on the $\mathrm{C}$ slot"). Moreover, the game sheet can cover some of the slots on the board to simplify challenges for first graders (instead of having an $\mathrm{N} \times \mathrm{N}$ board, they will be using a sheet just with some paths available -or even just one, for trivial challenges-).

Once the kid has understood the story, identified the blocks to use, and the path to follow, she is able to start placing the blocks on the board. To accomplish that mission, she has to take into account which numbers and letters are involved and place the blocks. In this example, blocks are similar to water pipes, some of them straight and some of them bent. These blocks have a small extension on their bottom, meant to be placed on a slot on the board. When kids place a block on a slot, the led placed on the top left side of the slot is turned on. The color of the LED is used to provide feedback: red when the position is wrong and green when it is right. The whole board will turn its LEDs red if the blocks chosen are wrong. As can be seen, Flow Paths provides immediate feedback to encourage self-discovery. Kids start trying until a green light is shown, and then they proceed with the following block. Once all the blocks are correctly placed on the board, the full path is turned on in blue color for a few seconds to simulate the water flow.

Example 2: Electronic Flows. This Flow Paths game is designed for high-school and higher-education students. Similarly to the previous example, this Flow Paths game uses a set of game sheets to provide a brief explanation about the goal of the game and to cover some of the slots of the board to place the blocks. The most relevant blocks of this game are those representing logic gates (e.g., AND, OR, NOT, XOR, etc.) and digital inputs (1 or 0$)$, because the general procedure of this set of challenges is to get the desired output using the appropriate setup of logic gates.

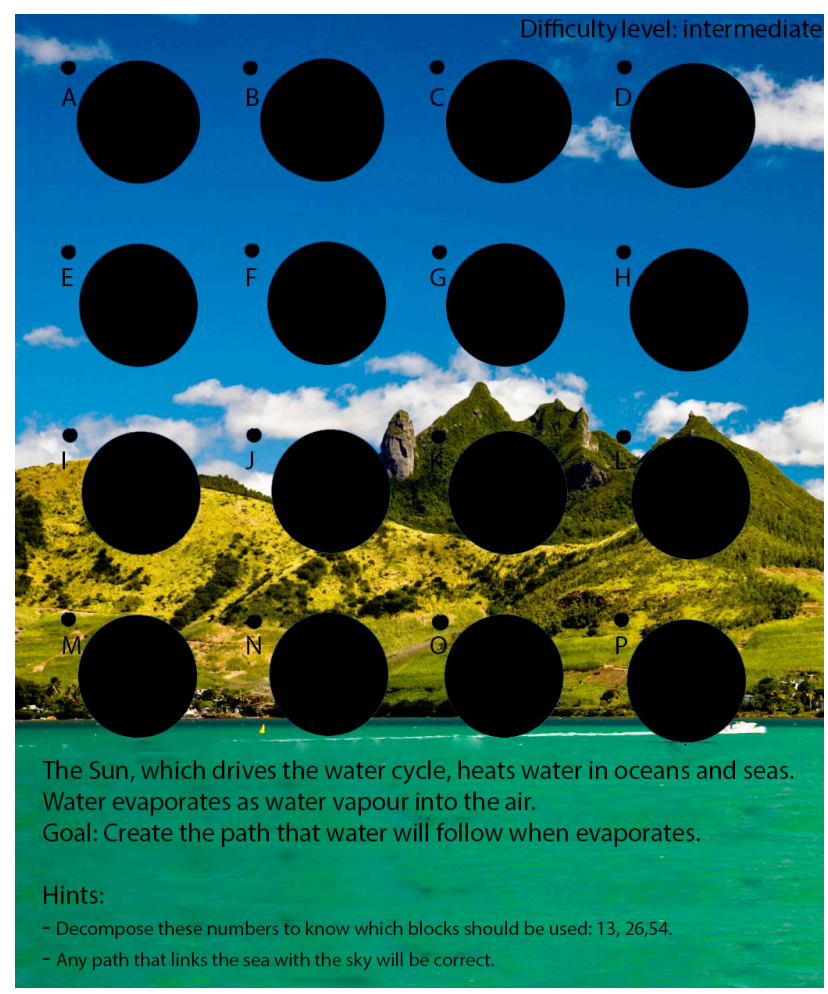

Figure 3. A Flow Paths game sheet designed for early-stages students.

The available set of game sheets provides digital electronics challenges with several levels of difficulty (see Fig. 4). In each game sheet there is a brief explanation of the goal of the challenge, background information (e.g., description about how some logic gates work), and hints about which blocks are involved. Instead of using numbers to identify the blocks like we did in the previous example, logic gates blocks are labeled with fake serial numbers, simulating the way electronic components are identified in the real world.

Regarding the feedback during the game play, placing blocks on incorrect slots does not make any LED to be turned on. Instead, the red LED of each block labeled with a 0 is turned on when placed on the board. The same happens with the green LED of each block labeled with a 1. Once those input blocks are connected with logic gates blocks, a red or green light path is created from the input blocks to the final block (the result block). Therefore, gamers can see what is going on the whole process and not only focus on the result.

\section{CONCLUSIONS AND FUtURE WORK}

In the 19th century, progressive education supporters tried to renew the education curriculum, going from a traditional teacher-centered methodology to studentcentered and task-based approaches $[9,10,11]$. Year later, Montessori proposed an educational philosophy based on the following principles: a) students should be placed at the center of school activities, b) teachers should act as helpers in learning events, situations and processes, and c) the school should be a prelude to the inclusion of students in social life. Montessori stated that an optimal learning experience requires autonomy, competence and relatedness of the learner [1]. Therefore, she assigned a 


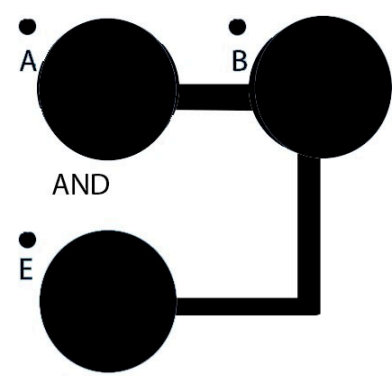

Difficulty level: easy.

Identify an AND gate block an place it on the $B$ slot. Use the test blocks to see how it works.

Hint: All inputs must be on to turn on an AND gate.

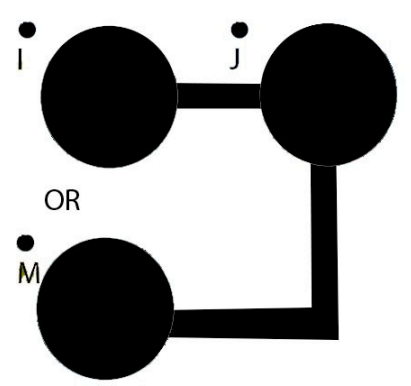

Identify an OR gate block an place it on the $B$ slot. Use the test blocks to see how it works.

Hint: At least one input must be on to turn on an OR gate.

Figure 4. A Flow Paths game sheet designed for high-school and higher-education students.

key role to physical objects because they allow selfdirected and purposeful learning activities [4, 12], and enable collaborative experiences in shared contexts with adults [13] or peers [14]. As we mentioned before, Flow Paths materials also allow self-directed and purposeful learning activities that can be shared with peers. Moreover, they are self-correcting and provide immediate feedback, can promote multi.-sensorial interactions (e.g., visual, auditory, and haptic) and are grouped by difficulty. Although most Montessori supporters prefer using lowtech approaches, learning materials made using Flow Paths follow Montessori's main goal: to explode the potential of each learner to improve autonomously in a structured environment.

Flow Paths is a standalone system that can be entirely manipulated by learners for learning concepts related to flows and paths. Its modular architecture allows extending its dimensions, adapt the difficulty of the challenges, and vary the scenario game easily. The design choices made to develop this system provide several advantages from an educational point of view. First, the use of physical blocks instead of virtualized objects enables the development of fine motor skills in early childhood of special needs learners. Second, these blocks can be handled without extreme care, since all active components of the system are placed on the board. Third, the independence of the system from a personal computer or mobile device (smartphone, tablet, etc.) reduces the need for adult intervention to use it. Learners can activate the board, interact with blocks and change scenario sheets by physically acting on the system. If the design of games developed on Flow Paths is properly scaffolded, there are extensive opportunities for self-learning. However, we think that educational games designed to test Flow Paths do not cover all capabilities of a system of this kind yet. Therefore, our future efforts are aimed at improving and expanding these educational activities, as well as testing and studying the learning processes that occur when using them.

\section{REFERENCES}

[1] Montessori, M. (1912). The Montessori Method. New York: Frederick Stokes Co.

[2] Lillard, A., Else-Quest, N. (2006). The early years: Evaluating Montessori Education. Science 29, September 2006: Vol. 313. no. 5795, pp. 1893-1894.

[3] Ullmer, B. and Ishii, H. (2000). Emerging frameworks for tangible user interfaces. IBM Systems Journal, 39(3\&4), 915-931. http://dx.doi.org/10.1147/sj.393.0915

[4] O'Malley, C. and Fraser, D.S. (2004). Literature review in learning with tangible technologies. NESTA futurelab report 12 , Bristol, 2004.

[5] Greenberg, S. and Fitchett,C. (2001). Phidgets: easy development of physical interfaces through physical widgets. In User Interface Software and Technology, 2001, pp. 209-218. http://dx.doi.org/10.1145/502348.502388

[6] Verhaegh, J., Fontijn, W., and Hoonhout, H. (2007). Tagtiles: optimal challenge in educational electronics. In TEI '07: Proceedings of the 1st international conference on Tangible and embedded interaction. New York, NY, USA: ACM, 2007, pp. 187-190. http://dx.doi.org/10.1145/1226969.1227008

[7] Hochenbaum, J. and O. Vallis. (2009). Bricktable: A Musical Tangible Multi-Touch Interface. In Proceedings of the Berlin Open. 2009. Germany.

[8] Iwata, T., Yamabe, T., Polojärvi, M., and Nakajima, T. (2010). Traditional games meet ICT: a case study on go game augmentation, In Proceedings of the fourth international conference on Tangible, embedded, and embodied interaction, January 24-27, 2010, Cambridge, Massachusetts, USA. http://dx.doi.org/10.1145/1709886.1709933

[9] Dewey, J. (1998). Experience and education. Kappa Delta Pi.

[10] Piaget, J (1953). How children form mathematical concepts. Scientific American, 189(5), 74-79. http://dx.doi.org/10.1038/ scientificamerican1153-74

[11] Bruner, J (1966). Toward a Theory of Instruction. New York: WW Norton.

[12] Papert, S (1980). Mindstorms: Children, Computers and Powerful Ideas. Brighton: Harvester.

[13] L. Vygotsky. Mind in society. Harvard University Press, Cambridge, MA, 1978.

[14] O'Donnell, A. M; A. King (1999). Cognitive perspectives on peer learning. Lawrence Erlbaum. ISBN 0805824480.

\section{AUTHORS}

A. Barredo is with the DeustoTech Institute at University of Deusto, Bilbao, Spain (e-mail: abarredo@opendeusto.es).

P. Garaizar is with the DeustoTech Institute at University of Deusto, Bilbao, Spain (e-mail: garaizar@deusto.es).

This article is an extended and modified version of a paper presented at the EDUCON2015 conference held at Tallinn University of Technology, Tallinn, Estonia, 18-20 March 2015. Manuscript received 20 April 2015. This work was supported in part by the Cátedra Telefónica - Deusto, Spain. Submitted 20 April 2015 Published as submitted by the authors 20 August 2015. 